\title{
Crisis de deuda soberanas: Aproximaciones en torno al debate contemporáneo de los mecanismos de reestructuración
}

\section{Crise da dívida soberana: Abordagens ao debate contemporâneo dos mecanismos de reestruturação}

Recebido em: 02 de abril de 2018

Aprovado em: 04 de julho de 2018

DOI: 10.5752/P.2317-773X.2019v7.n1.p7

José Marcelino Fernández Alonso ${ }^{1}$

\begin{abstract}
1. Doctor en Relaciones Internacionales. Investigador asistente del Consejo Nacional de Investigaciones Científicas y Tecnológicas (CONICET). Docente de Economía Internacional y Finanzas Internacionales, Facultad de Ciencia Política y Relaciones Internacionales, Universidad Nacional de Rosario. Rosario/Argentina. ORCID: 0000-00020260-6802 (1D)
\end{abstract}

\begin{abstract}
RESUMEN
Como consecuencia de las modificaciones político-estructurales acarreadas por la globalización, las crisis de deuda soberana se repitieron con mayor frecuencia. En este contexto, se entretejió un prolífico debate respecto al modo más eficiente para sortear las externalidades derivados de la contrariedad de un Estado para afrontar sus compromisos financieros externos. No obstante la pluralidad de perspectivas y proyectos, las deliberaciones sobre la temática se restringieron a la confrontación de dos formulaciones: por una parte, la propuesta "estatutaria"; por la otra, la "contractual". La reticencia de Estados Unidos y de los principales agentes del sistema financiero internacional a la visión estatutaria -impulsada originalmente por funcionarios del Fondo Monetario Internacional-, hicieron que en 2003 las discusiones parecieran clausurarse a favor del proyecto contractualista. Sin embargo, la sucesión de crisis de deuda soberana que dieron desde entonces y los reveses de la Argentina en las demandas entabladas por tenedores de instrumentos de títulos públicos en default renuentes a participar en el proceso de reestructuración hizo resurgir el debate, propiciando reformulaciones institucionales. El propósito del presente artículo es analizar el estado del debate contemporáneo sobre los mecanismos de reestructuración de deuda soberana. Dicho análisis es realizado a partir de los aportes del institucionalismo liberal. Se entiende que el abordaje de estas discusiones resulta de vital importancia en consideración del crecimiento exponencial del nivel de endeudamiento de los Estados -de los denominados "emergentes", en particular- durante los años recientes.
\end{abstract}

Palabras clave: Crisis de deuda soberana - Mecanismos de reestructuración Nueva arquitectura financiera internacional

\section{ResUmo}

Como resultado das mudanças político-estruturais provocadas pela globalização, as crises da dívida soberana eram repetidas com maior freqüência, afetando cada vez mais um número maior de atores e tornando-se cada vez mais difícil de administrar. Neste contexto, um prolífero debate foi interligado com respeito à maneira mais eficiente de superar as externalidades derivadas da contrariedade de um Estado em enfrentar seus compromissos financeiros externos. Apesar da 
2. Con propósito de clarificar los conceptos recién referidos, se pone de manifiesto en primer término que el trabajo se asiste de la definición de Kevin Gallagher en lo concerniente a la noción de crisis de deuda soberana. De acuerdo con el autor, una crisis de deuda soberana da cuenta de "aquellas

situaciones en las que un Estado no puede -o no quiere- simplemente hacer

frente a sus compromisos financieros externos" (GALLAGHER, 2011, p. 1). En tanto, por cesación de pagos o default, el trabajo se vale de las consideraciones de Chukwu (2011, p. 51), quien la define

como una alteración en el cumplimiento de las obligaciones financieras asumidas por un Estado en uno o varios instrumentos de deuda, el cual puede manifestarse como una suspensión de

pagos de interés o de intereses y e capital, la modificación de las fechas

de pago, y/o la declaración de una moratoria sobre el total de la deuda o sobre una porción de la misma. En consideración de lo antedicho, la cesación de pagos no es sino una de las posibles derivaciones que puede de tener una crisis de deuda soberana.

3. Conforme se anuncia en su sitio web (http://www.clubdeparis.org), el "Club de París" es un grupo informal de acreedores estatales -la gran mayoría, miembros fundadores de la Organización

para la Cooperación y el Desarrollo Económicos (OCDE)- surgido a mediados

de los años 50 con el propósito de renegociar deudas en situación irregular que la República Argentina mantenía por entonces con ciertos países desarrollados. Desde su institucionalización, ha devenido un espacio de reestructuración de deudas interestatales en decenas de oportunidades. En la actualidad, cuenta con 19 miembros permanentes y una Secretaría administrativa en París la cual coordina las actividades del colectivo. En términos generales, el "Club de París" acuerda "minutas" o patrones de negociación que deben ser observadas en las negociaciones bilaterales particulares el Estado deudor y sus pares acreedores. Para un análisis en profundidad sobre el mismo, se sugiere Cosio-Pascal (2008).

4. En este punto, se remarca que diversos son los estudios históricos que

dan cuenta de estas discusiones respecto a los instrumentos institucionales orientados a enfrentar crisis de deuda soberana. Además de los trabajos ya citados de Winkler (1933); Sturzenegger y Zettelmeyer (2007) y Brooks y Lombardi (2014), se destacan los de Kindleberger

(1978), Lipson (1985); Tomz (2007): Waibel (2011) y Wright (2012). pluralidade de perspectivas e projetos, as deliberações sobre o assunto restringiram-se na prática ao confronto de duas formulações apresentadas em uma chave dicotômica: de um lado, a proposta "estatutária"; por outro, o "contratual". A relutância dos Estados Unidos e atores-chave do sistema financeiro internacional para a visão legal originalmente -driven por funcionários do Fundo Monetário Internacional, feita em 2003 parecem próximas das discussões para o projeto contratualista. No entanto, a sucessão de crises de dívida soberana que ocorreu desde então e reveses da Argentina nos processos movidos por detentores de títulos públicos padrão relutantes em participar no processo de reestruturação ressuscitou o debate, incentivando reformulações institucionais. O objetivo deste artigo é analisar o estado do debate contemporâneo sobre os mecanismos de reestruturação da dívida soberana. Esta análise é realizada com base nas contribuições do institucionalismo liberal. Entende-se que a abordagem dessas discussões é de vital importância, tendo em vista o crescimento exponencial do nível de endividamento dos Estados - em especial dos chamados "emergentes" - nos últimos anos.

Palavras-chave: Crise da dívida soberana - Mecanismos de reestruturação Nova arquitetura financeira internacional

Introducción

A semejanza de los fenómenos que los motorizaron históricamente, los debates en torno a la gestión de las crisis de deuda soberana -los defaults, como expresión más recurrente de ellas- resultaron una constante en el discurrir del sistema westfaliano, asumiendo intensidad incremental en los últimos decenios en concomitanciaala expansión e intensificación del fenómeno de la globalización ${ }^{2}$. Conforme ilustran Das, Papaioannou y Trebesch (2012), entre 1950 y 2010 las reestructuraciones de deuda soberana -a la postre el mecanismo dominante en la resolución de crisis de endeudamiento público-, totalizaron más de 600 casos abarcando 95 países. Dentro de ese universo de casos registrados, 186 fueron con acreedores privados (bancos extranjeros y tenedores de bonos) y 447 fueron acuerdos de reestructuración en el marco del Club de París ${ }^{3}$.

Tal como Skylar Brooks; Domenico Lombardi (2014) alertan, los desarrollos y desenlaces de cada uno de los debates en torno a la gestión de trances de endeudamientosoberano reflejaron -y reforzaron- los principales caracteres del contexto internacional circundante, al acarrear la (re)articulación de una multiplicidad de procedimientos institucionales para hacerles frente ${ }^{4}$. En efecto, de pasarse de soluciones basadas en el uso o amenaza de uso de la fuerza en las etapas primarias del sistema westfaliano, las respuestas a la problemática de marras pasaron a canalizarse crecientemente mediante arreglos institucionales ajustados al Derecho ${ }^{5}$. Esta identidad entre los debates respecto a la gestión de la crisis de deudas soberanas y las particularidades del contexto internacional no resulta nadaantojadiza si se considera que las crisis de deuda soberana ponen endiscusión uno de los principiosestructurante de todo orden social: la delimitación y defensa de la propiedad privada.

Sin perjuicio de lo antedicho, debe reconocerse que la naturaleza anárquica propia de la arena internacional -entendida en simples términos como la ausencia de una autoridad supranacional- impone ciertas peculiaridades a las crisis de deuda soberana y a los debates en torno al 
modo de encararlas, por extensión. En este sentido, se apunta en primer término que a diferencia de lo que sucede en el ámbito nacional/doméstico en el que las dificultades de una firma o agente económico pueden canalizarse mediante procedimientos de quiebras o bancarrota, el ámbito internacional adolece de un mecanismo de enforcement efectivo ante un episodio de cesación de pagos de un Estado soberano. Por otra parte, "aunque los contratos de deuda soberana sean difíciles de cumplir, lo cierto es que también duran para siempre. Sin un procedimiento de quiebra, la deuda pública no puede ser liquidada y darle al Estado en crisis "un nuevo comienzo"6 (BUCHEIT et. al., 2013, p. 15, traducción propia).

En los últimos decenios, el debate sobre los dispositivos institucionales para la gestión de episodios de cesación de pagos y crisis de deuda soberana, en general, se inscribieron -e inscriben aún, dado su carácter irresoluto- dentro un debate de orden sistémico, el cual fue gestado en concomitancia con el estallido de la sucesión de colapsos económico-financieros de mediados de los años '90. Dicho debate, denominado genéricamente como el de la "Nueva Arquitectura Financiera Internacional" (NAFI), se erigió sobre un amplio -e inaudito- consenso entre actores de diversa índole y posicionamientos político-ideológicos respecto a la necesidad de ajustar las estructuras normativo-institucionales del sistema financiero internacional a las dinámicas globales de hogaño. Dado su carácter sistémico, las discusiones de la NAFI comprehendieron problemáticas tan diversas como complejas, tales como el rol de las instituciones financieras internacionales (IFI), la sustentabilidad de los regímenes cambiarios, el control de los movimientos de capitales, las regulaciones a las agencias calificadoras de riesgo, entre otras?

En lo que respecta a las deliberaciones específicas sobre los mecanismos y/o dispositivos para la resolución de episodios de cesación de pagos soberanos, se advierte que las mismas giraron en torno a la idea de que los cambios experimentados durante las últimas décadas en los mercados internacionales de deuda pública imponían -imponen- desafíos muy diferentes a los conocidos en otrora, cuando el financiamiento de los Estados dependía excluyentemente de los recursos provistos por otras entidades soberanas, organizaciones multilaterales o la banca internacional (LI, 2013, p.1). Así pues, los protagonistas o interlocutores del mentado debate asumieron cual premisa de partida que el crecimiento y la complejización de los mercados de deuda soberana reportados a raíz de la incorporación de un sinnúmero de agentes heterogéneos, atomizados y dispersos en todo el mundo obligaba a ensayar canales o mecanismos institucionales alternativos a los instaurados décadas atrás: el denominado "Club de París" (para la renegociación de deudas interestatales) y el "Club de Londres" (para aquellas otras asumidas con la banca privada internacional), por ejemplo ${ }^{8}$.

Numerosas fueron las propuestas que se alumbraron en el marco del debate por la NAFI en su capítulo dedicado a los mecanismos de reestructuración de deuda soberana. No obstante esta pluralidadproyectos-los cuales incluyeron la adopción de códigos de conducta entre los Estados y acreedores ${ }^{9}$ o la constitución de una corte internacional para quiebras de deudas soberanas ${ }^{10}$-, lo cierto es que en la práctica las deliberaciones sobre la temática se restringieron a la confrontación de dos formulaciones
5. Habida cuenta de su abigarrado historial en crisis de deuda soberana, los Estados latinoamericanos -la República Argentina, en particular- asumieron un rol protagónico dentro de estas discusiones, siendo la formulación de la denominada Doctrina Drago a inicios del siglo XX con su cuestionamiento a la pretensión de cobro de acreencias de potencias europeas a Venezuela vía coerción una de las manifestaciones más palmarias de ello.

6. En el texto original, "although sovereign debt contracts are hard to enforce, they also last forever. Without bankruptcy, sovereign debt cannot be discharged to give the country a fresh start".

7. Para la profundización analítica sobre el debate de la NAFI se recomienda los siguientes textos: Bustelo (2000); Akyuz (2002); Villanueva (2003); Underhill y Zhang (2003) y Ocampo (2014).

8. El "Club de Londres" refiere a un grupo informal de entidades bancarias internacionales que albergó negociaciones de deuda entre éstas y Estados soberanos. A diferencia del mencionado en primer término, el "Club de Londres" no cuenta con miembros permanentes ni secretaría. En este punto, se remite a Reiffel (2003).

9. Al respecto, pueden verse los trabajos consignados a continuación: Institutefor International Finance (IIF) (2004), y Couillault y Weber (2003).

10. En este punto, se sugiere consultar los trabajos de Stiglitz (2003); Reinisch (2003) y Ugarteche y Acosta (2005). 
11. De acuerdo con la premisa de los referidos internacionalistas, "las instituciones internacionales (...) varían en diversas dimensiones. La OMC y el régimen internacional de protección de los osos polares son ambas instituciones, pero difieren en lo que refiere al alcance de sus reglas, los recursos disponibles para la organización formal y el grado de diferenciación burocrática (...)" (GOLDSTEIN et al., 2001,p.3).Para los autores, son tres las dimensiones a ponderar: la obligatoriedad, la precisión y la delegación. En lo que respecta a las propuestas de reestructuración de deuda en particular, el acento está puesto en la última dimensión, entendida como la resultante de la decisión de conferir a una tercera parte la autoridad para "implementar, interpretar y aplicar aquellas reglas; resolver disputas y realizar (posiblemente) nuevas reglas" (ABBOT et al., 2001, p.17). presentadas en clave dicotómica: por una parte, la propuesta "estatutaria" diseñada y fomentada originalmente por el staff del Fondo Monetario Internacional (FMI) entre 2002 y 2003; por la otra, la "contractualista", impulsada por entonces el Subsecretario del Tesoro norteamericano para Asuntos Internacionales, John B. Taylor. Sin ánimo ni posibilidades de detallar en este espacio introductorio las directrices de ambos abordajes, se limitan estas líneas a apuntar que la propuesta estatutaria propugna que las operaciones de reestructuración de deuda soberana sean administradas por un organismo internacional específico -el FMI, por ejemploconforme la letra de una convención o tratado internacional específico (HOFFMAN, 2014; GIANVITI et Al., 2010). La posición contractualista, por contraste, entiende que los procesos de reestructuración deben de conducirse conforme lineamientos -cláusulas, concretamente- establecidos en los propios contratos de deuda soberana.

Si bien en el promediar de la primera década del siglo XXI, la última solución pareció imponerse sin más, el estallido de crisis de deuda soberana en la periferia de la Eurozona, primero y Estados de menor gravitación en el escenario financiero global (Ucrania y Puerto Rico), como asimismo la continuidad -y agravamiento aún- de la denominada "saga" de la República Argentina con sus acreedores internacionales en la justifica norteamericana hicieron reflotar el mentado debate en los años recientes. Las advertencias del FMI y de las agencias calificadoras de riesgo a propósito del exponencial crecimiento experimentado por los mercados de deuda soberana durante los últimos años avivan de seguro la vigencia del debate (FMI, 2018; DOBBS et. al., 2015).

En recurso de las teorizaciones de Judith Goldstein, Miles Kahler, Robert Keohane y Anne-Marie Slaughter respecto a los diferentes esquemas de institucionalización en la arena internacional, es dable señalar que todas las propuestas de gestión de crisis de deuda soberana involucradas en el debate de la NAFI pueden ser ubicadas dentro de un continuum conforme el nivel de delegación propugnado en cada una de ellas ${ }^{11}$. Al propugnar esquemas de delegación contrapuestos, las propuestas estatutaria y contractualista recién consignadas pueden identificarse efectivamente cual extremos: por un lado, un modelo plenamente delegativo, por el otro, un modelo liberal, renuente a resignar soberanía por parte de los Estados. Siguiendo las contribuciones de los mencionados referentes del institucionalismo liberal, puede señalarse pues que el enfrentamiento de posiciones respecto al nivel de delegación en los mecanismos de gestión de crisis de deuda soberana supone una manifestación de un debate mucho más profundo y abarcativo respecto al diseño y eficacia de las instituciones en la esfera internacional. En este sentido, se sostiene que detrás de cada una de las propuestas inherentes a la gestión de crisis de deuda soberana pueden vislumbrarse lógicas racionales orientadas a la búsqueda racional de agenciar (o preservar) recursos simbólicos y/o materiales.

El propósito del presente artículo es analizar el estado del debate contemporáneo sobre los mecanismos de reestructuración de deudas soberanas. En consideración de ello, el artículocomienza por desentrañar las principales directrices sostenidas por las propuestas que mayor impacto político-económico tuvieron en marco del debate desde principios de 
siglo XXI. Con tal fin, identifica los promotores de cada de ellas y los argumentos que esgrimieron en torno a los tres problemas que condicionan a todos los procesos de reestructuración de deuda soberana, conforme explica Schwarcz (2004, p.1992, traducción propia): "el problema de los holdouts $^{12}$ o el de la acción colectiva, el problema del riesgo moral y el problema de los ciudadanos/pagadores de impuestos llamados a sustentar los procesos de "salvataje" y/o reestructuración"13. Una vez contextualizado los lineamientos y actores que impulsaron cada una de las posiciones del debate en torno a los mecanismos de reestructuración en el debate a principios de siglo XXI, el artículo avanza en las derivaciones que cada una de ellas tuvieron en los años recientes y en los días actuales. Por último, la presente producción culmina con unas reflexiones conclusivas.

El debate entre las propuestas "estatutarias" y "contractualistas" en los primeros años del siglo xxi: a propósito de sus orígenes y su cierre precipitado

Tal como se adelantó líneas arriba, la sucesión de crisis experimentada durante el último lustro del siglo XX incitó la articulación de un inusitado y comprensivo debate en torno a las estructuras y dinámicas del sistema financiero internacional, asumiendo a la problemática de la gestión de las crisis de deuda soberana como uno de sus capítulos troncales. Estas discusiones sobre el particular reportaron su punto más álgido en los meses inmediatos posteriores a la declaración de cesación de pagos anunciada por la República Argentina el 23 de diciembre de 2001. A resumidas cuentas, el default más importante en volumen en la historia del sistema financiero global, todo lo cual le valió que fuera calificado como "la madre de todas las crisis de deuda soberana” (ROUBINI y SESTER, 2004).

De acuerdo con lo explicado por Carlos Alfaro (2003), laspropuestas estatutariasde reestructuración de deudas soberanas descansan sobre dos presupuestos centrales: por una parte, la existencia de un tratado o convención internacional que delimite el protocolo a seguir ante un episodio de crisis de deuda soberana y, por otra parte, la instauración y/o definición de un organismo internacional encargado de administrar los procedimientos allí consignados. Sobre la base de estos presupuestos, se postularon en el transcurrir de los años en los que discurrió el mentado debate de la NAFI una variedad de propuestas.

Sin dudas, la iniciativa que mayor repercusión dentro de este esquema tuvo fue la propulsada por el propio FMI entre noviembre de 2001 y abril de 2003 a través de Anne Krueger, Subdirectora gerente y Economista principal del organismo por entonces. Denominado primigeniamente como "Mecanismo de Reestructuración de Deuda Soberana" (MRDS), el proyecto preveía la instauración de un esquema estatutario que posicionaba al propio Fondo en el rol capital de la gestión y/o administración de los episodios de reestructuración. En este sentido, la iniciativa estipulaba que los Estados en dificultades acudiesen al organismo a los fines de solicitar asistencia ante la inminencia de una crisis de deuda soberana. En dicho contexto, el país recibiría pues financiamiento extraordinario por parte del FMI y de eventuales agentes privados a condición de que implementase un programa económico que le permitiese sortear las contrarie-
12. Este término, proveniente del idioma inglés, remite a la agregación de dos palabras, a saber: el verbo to hold (mantener) y el adjetivo out (afuera). En breve, la alocución refiere a "los que se mantienen afuera" (del canje de deuda, por caso). Dicho esto, se indica que la identificación de los holdouts en los procesos de reestructuración como derivación prototípica de un problema de acción colectiva cuenta con numerosas referencias entre la literatura especializada. Al entender de este autor, una de las explicaciones más acabadas sobre el particular se encuentra en un trabajo de Kentaro Tamura. Según el precitado cientista, "el fenómeno de los holdouts es una de las expresiones más significativas del problema de la acción colectiva, manifestada muy particularmente en el denominado "dilema del prisionero" de la teoría de los juegos.

13. En el texto original: "Sovereing debt restructuring gives rise to three distinct problems: the holdout, or the collective action, problem; the moral harazd problem; and the taxpayer-funding problem". 
dades que habían conducido a la crisis e iniciase prontamente el proceso de negociación con sus acreedores.

Del mismo modo, y en ánimo de sortear uno de los argumentos más recurrentes de los detractores de los rescates financieros durante los años precedentes, el proyecto bajo análisis señalaba que el gobierno del país asistido debía comprometerse a implementar medidas que refrenaran la salida de capitales (KRUEGER, 2002, p. 27). Dicho en otros términos, el proyecto buscaba sortear los cuestionamientos de políticos y académicos posicionados en diversos sectores del espectro ideológico-esto es, desde la izquierda y el progresismo hasta la derecha (neo)liberal- respecto al hecho de que los capitales habilitados por el organismo a un país devenido en crisis fueran prontamente dilapidados en procesosde fuga, sumiendo a todos los actores intervinientes enel rescate en una situación peor a la conocida previo el inicio de la crisis. En esta línea argumental, se inscribían las críticas de funcionarios de la administración Bush respecto a que cada rescate implicaba un derroche los recursos de los ciudadanos de los principales socios del Fondo, de Estados Unidos, en particular.

Si bien en todos sus escritos y exposiciones públicas, la Subdirectora del FMI insistía que la institución se abstendría de intervenir en las negociaciones entre el Estado en crisis y sus acreedores, el organismo habría de supervisar las variables macroeconómicas del primero, garantizando consiguientemente la buena fe del mismo. En consideración del ya mencionado problema de los holdouts y sus posturas obstruccionistas, el MRDS planteaba que durante el período en que se prolongara la vigencia del mecanismo, quedaba limitada la posibilidad de activar demandas judiciales (EICHENGREEN, 2003).

Por otra parte, una vez alcanzado un acuerdo con la aprobación de una mayoría calificada de acreedores, tal decisión devendría obligatoria para todos los acreedores (BUSTELO, 2005). En caso de que el proceso de negociación no redundase en un acuerdo aprobado por una mayoría calificada, los acreedores tendrían la oportunidad de ventilar sus controversias en un órgano a instaurar dentro del FMI para tal fin. En busca de resguardar la imparcialidad entre los actores en pugna, el proyecto de marras marcaba que la nueva instancia de resolución de controversias debía gozar de independencia frente a los referentes de la Dirección, el Directorio Ejecutivo, el staff técnico-burocrático y de los propios Estados miembros del Fondo. De este modo, pues, el proyecto presentado por Krueger procuraba refrenar las críticas formuladas por sus detractores respecto al eventual cruce de intereses del propio FMI.

Llegado este punto, se impone oportunosubrayar que la implementación del proyecto requería la enmienda de diversos artículos del Convenio Constitutivo del organismo al implicar ajustes tanto en términos funcionales comoburocrático-estructurales. A propósito de ello, resulta pertinente rememorar que conforme la letra del mencionado instrumento, toda modificación de tal clase debe de contar con al menos el $85 \%$ de los votos del organismo, lo que en términos fáctico imponía la venia de Estados Unidos, circunstancia no habilitada por entoncesen consideración del rechazo de los funcionarios de la administración presidida por George W. Bush a la propuesta. 
Recipiendario de críticas de vastos referentes políticos y académicos -de John B. Taylor, entonces Subsecretario del Tesoro para Asuntos Internacionales y principal defensor del enfoque contractual-, el proyecto fue progresivamente perdiendo respaldos hasta ser completamente desistidopor el FMI en abril de 2003. En este marco, y tal cual lo adelantado líneas arriba, el debate pareció saldarse a favor de la perspectiva contractualista.

Diversas fueron las interpretaciones realizadas por los estudiosos del problema bajo análisis para dar cuenta de rechazo de Estados Unidos a la iniciativa del FMI y el impulso consiguiente de la salida contractual o "de mercado". Según el análisis de Anna Gelpern; Ben Hellery; Brad Setser (2016), el debate en torno a los mecanismos institucionales de administración de crisis de deuda soberana no pudo escapar de una lectura estado-céntrica propia de un país con gravitación decisional en el ordenamiento político-económico global, y de las finanzas internacionales, en particular. En sus palabras, "para la mayoría de los funcionarios de EEUU, la idea de que un tratado pudiera prevalecer sobre los contratos financieros suscriptos bajo la ley de Nueva York o de que un organismo internacional podría prevalecer sobre los tribunales norteamericanos era simplemente inaceptable" (Gelpern, et. al., 2016, p. 116).

Por otra parte, Skylar Brooks; Eric Helleiner (2017), acentúan que el proyecto estatutario recibió rechazos generalizados debido a que limitaría la influencia "de facto" de EEUU y de otras potencias occidentales en el manejo de las crisis de deuda al delegar a una nueva autoridad internacional la decisión de si un país afectado por una crisis debería someterse a una reestructuración de la deuda. Adicionalmente, el rechazo de EEUU al proyecto estatutario no podría desprenderse del ánimo de preservar el poder para diseñar y velar por el cumplimiento de los contratos de deuda soberana suscriptos y emitidos bajo sus leyes. Por cierto, poder compartimentado con el Reino Unido, habida cuenta de la primacía de las plazas de Nueva York y Londres dentro de la estructura y dinámica de los mercados de deuda soberana. Por último, y siguiendo con el análisis de los autores recién mencionados, no puede disociarse la resistencia norteamericana al proyecto del MRDS de los lineamientos político-ideológicos de los decisores de mayor peso de la administración Bush Jr. (HELLEINER, 2005). Por cierto, lineamientos que en su capítulo externo abrevaba en las conclusiones críticas de la denominada "Comisión Meltzer" al rol de "prestamista de última instancia" asumido por el FMI desde la década del ochenta y sus externalidades negativas sobre el "riesgo moral"14. En correspondencia con lo expuesto por Schwarcz(2004), la cuestión del riesgo moral no resulta menor en la problemática de las crisis de deuda ya que insta a los agentes económicos a asumir comportamientos temerarios al descontar de la existencia de una salvaguarda, un rescate del FMI, por caso, financiado de acuerdo con el imaginario y discurso de los funcionarios y académicos promotores de los resultados de la mencionada Comisión- por los ciudadanos de los principales socios del organismo, de Estados Unidos, en particular. Por último, y conforme marca Ugo Panizza (2013), la identificación de los rescates del FMI como mecanismos generadores de riesgo moral y de comportamientos disruptivos en el sistema financiero internacional podía bien graficarse en la ocurrencia de Robert Barro de reemplazar el nombre
14. La Comisión Meltzer fue conocida así en alusión el economista que la presidiera, Allan Meltzer. Formalmente, se denominó Comisión Asesora de Instituciones Financieras Internacionales. Fue instaurada por el Comité Económico Conjunto del Congreso de Estados Unidos en 1998 y emitió su informe en 1999 (www.house.gov/jec/imf/meltzer. $\mathrm{htm})$. La continuidad de las conclusiones y líneas discursivas de los funcionarios de la administración de Bush quedan ilustradas en las declaraciones del Secretario del Tesoro Paul O'Neill en el Foro Económico Mundial, el 1 de febrero de 2002, disponible en: www.treas.gov/ press/releases/po974.htm. 
del organismo -y su sigla en voz inglesa, por lógica extensión- por el de "Instituto de Riesgo Moral" (IMH) (PANIZZA, 2013, p. 7).

Tal como señala Hoffman (2014, p. 13), comparado con el estatutario, la concreción del modelo contractual resultaba menos ambiciosa, y por tanto, menos gravosa en términos de costos político-institucionales para los actores más gravitantes del sistema financiero internacional. Tal cual lo anunciado en la introducción, el ensayo contractual postula la mera incorporación de determinadas cláusulas en los contratos de deuda soberana sometiendo la administración de los procesos de reestructuración a la observancia de los términos pactados originalmente en el mercado entre los Estados y sus acreedores internacionales.

En consideración del andamiaje teórico-conceptual sobre el que se estructura el presente trabajo, es de apuntar que el modelo contractual reniega de la posibilidad de delegar la administración de las crisis de deuda soberana a una institución internacional en particular,patrocinando por defecto la incorporación de cláusulas en los instrumentos de deuda que establezcan los procedimientos a seguirse ante la eventualidad de incumplimiento -la irregularidad en el pago de los compromisos derivados de los mismos, por ejemplo-. Si bien son varias las disposiciones que pueden incorporarse en los instrumentos de deuda a modo de "cláusula de acción colectiva" (CAC), las de mayor significación refieren a la de una "supermayoría" exigible de acreedores para que un Estado pueda modificar los términos originales de un título público. Por lo general, el piso de dicha "supermayoría” oscila entre el $75 \%$ y el $80 \%$. Es ésta, la respuesta primaria del ensayo bajo análisis al mencionado problema de los holdouts. Pero si bien esta respuesta procuraba sortear el rechazo de algunos tenedores de deuda de participar de toda propuesta de reestructuración, la incorporación de la CAC de supermayoría, alertan sus detractores, no ultima decididamente la eventualidad de una minoría con capacidad de bloqueo. Más aún, los críticos de las salidas contractualistas o liberales ponen énfasis que los tenedores reacios a avanzar en un proceso de reestructuración podrían de hacerse en el mercado secundario de instrumentos adicionales hasta alcanzar el porcentaje mínimo para obstruir todo ensayo de reestructuración.

Por otra parte, y tal como lo advierte Haley (2014), pese a que las CACs pueden facilitar la reestructuración de una serie particular de bonos, no habilitan una salida concreta para la totalidad de la deuda de un Estado en crisis ya que los incentivos para avanzar en una reestructuración no pueden ser necesariamente compartido por las mayorías de los demás instrumentos de deuda.

En este contexto, resulta importante destacar que además de la referida a la "supermayoría", destaca entre las CACs, la cláusula de "aceleración", la cual

$$
\begin{aligned}
& \text { "determina que, en el caso de no-pago de cualquier parte de una determinada } \\
& \text { serie de títulos de deuda externa re-estructurada, los acreedores podrán declarar } \\
& \text { inmediatamente vencidos el principal, los intereses y todos los demás valores } \\
& \text { adeudados sobre los títulos de deuda de aquella serie, es decir, se declararía toda la } \\
& \text { deuda de plazo vencido, aún cuando no esté "vencida" (BUCHIERI, 2014, p. 68). }
\end{aligned}
$$

En sintonía con lo alertado por Gelpern (2013), en tanto provisiones contractuales guiadas exclusivamente por el interés privado, las CACs carecen de lógica para comprehender y abarcar externalidades negativas de 
toda reestructuración como pueden ser los problemas distribucionales en la sociedad del Estado devenido en crisis o la equidad en las respuestas para con todos los afectados por la crisis. En este sentido, no se hace posible imponer consideraciones de mayor alcance -políticas, económicas, geoestratégicas, entre otras- a inversores regidos por el interés privado y amparado precisamente por las mentadas cláusulas. Ahora bien, estas evaluaciones esgrimidas por los detractores de las CACs resultaban contrapesadas por los argumentos centrales de las CACs: el evitar delegar a un tercero la administración de la reestructuración y, sobre todo, la implementación de mecanismos de financiamiento. Esto último colocaba a la salida de mercado inherente a las CACs como la menos gravosa en términos materiales. Conforme repetía Taylor en sus alocuciones, la adopción de CACs alejaba todo procedimiento de salvataje que debía ser sufragado por los ciudadanos de los principales países miembros del FMI.

En concordancia con Leonardo Stanley (2009), se recuerda que el uso de las CACs distaba de ser novedoso. Dichas cláusulas han sido utilizadas bajo bonos emitidos por legislación inglesa desde 1879. De hecho, y tal como explica Juan José Cruces (2016), existieron en bonos de ley estadounidense con tales cláusulas hasta que fueron prohibidas por el marco regulatorio de los bonos corporativos (Trust Indenture Act) en 1939. Las CACs volvieron a implementarse en el mercado de deuda bajo legislación norteamericana tras el año 2003, merced el impulso del modelo por el mencionado Taylor y una serie de recomendaciones de foros multilaterales -Grupo de los Diez- y del propio FMI una vez aceptada las limitaciones políticas para avanzar en el proyecto promovido por su vice directora gerente.

Siguiendo a lo señalado por Cruces (2016), es de advertir que "aunque se considera que México en 2003 fue la primera emisión moderna con CAC en Nueva York, en realidad hubo tres emisiones previas: el Líbano en 2000, Qatar en 2000 y Egipto en 2001” (CRUCES, 2016, p. 93). Desde entonces, los instrumentos de deuda emitidos en tal plaza contienen provisiones legales de este tipo.

Esta innovación institucional -la cual, en los hechos, sólo implicaba el abandono del criterio de unanimidad exigido por la legislación en Nueva York para modificar los términos originales de los instrumentos financieros- fue identificada como la piedra angular de un nuevo régimen internacional de abordaje de crisis de deuda soberana basado en criterios liberales o de mercado. Poco tiempo debió de transcurrir, no obstante, para que se explicitaran las deficiencias del mismo.

La reapertura del debate en la segunda década del siglo xxi: la reconsideración de las propuestas "estatutarias" y contractualistas tras las crisis de deuda de la eurozona y la república argentina

La renuncia del FMI a proseguir impulsando el MRDS y la emisión generalizada de títulos con CACs a partir de los primeros meses de 2003 supuso un cierre de facto del debate en torno a los mecanismos de administración de las crisis de deuda soberana. Conforme expresan Richard Gitlin; Brett House (2015), la relativa estabilidad del sistema financiero reportada en los años que siguieron a la clausura del mentado debate pa- 
15. Para esta cuestión en particular se recomienda los abordajes de Gelpern (2016) y Brooks; Helleiner (2017).

16. Originalmente, la medida comenzaría a regir a partir de junio de 2013. Empero, y conforme se avanzará luego, las urgencias del contexto condujeron a que los decisores del Eurogrupo adelantaran la vigencia de la misma seis meses antes.

17. En el comunicado de prensa emitido tras la mencionada reunión se expresó: "In the unexpected event that a country would appear to be insolvent, the Member State has to negotiate a comprehensive restructuring plan with its private sector creditors, in line with IMF practices with aview to restoring deb sustainability. If debt sustainability can be reached throughthese measures, the ESM may provide liquidity assistance. In order to facilitate this process, standardized and identical collective action clauses(CACs) will be included, in such a way as to preserve market liquidity, in the terms and conditions of all new euro area government bonds starting in June

2013." EU (2010) EUROPEAN UNION, "Statement by the Eurogroup», Disponible en: http://www.consilium.europa. eu/press/press-releases/2010/11/pdf/ Statement-by-the-Eurogroup/

18. Concretamente, el artículo 12.3 del mencionadotratadoreza: "Collective action clauses shall be included, as of 1 January 2013, in all new euro area government securities, with maturity above one year, in a way which ensures that their legal impact is identical." reció justipreciar a los promotores del enfoque contractualista imperante. Empero, el estallido de las crisis de deuda de la Eurozona y otras economías de la periferia global, como derivación inexcusable de la gran crisis hipotecaria-financiera desatada años antes, más la acumulación de reveses de la República Argentina en las demandas incoadas por sus acreedores en situación irregular expusieron las externalidades del régimen de mercado articulado por defecto -o "no régimen", tal como sostiene Panizza (2013)- y la persistencia del déficit institucional internacional en el particular. De este modo, el inicio de la segunda década del siglo XXI marcó el resurgimiento del debate entre "estatutarios" y "contractualistas", todo lo cual dio cuenta de lo precipitado que había sido la clausura del debate en su etapa anterior.

De acuerdo con lo expresado Brooks; Helleiner (2017), la prevalencia de la fórmula de mercado en 2003 procuró ser apuntalada mediante una serie de innovaciones institucionales parciales y/o identificadas en los márgenes del régimen internacional para la gestión de las crisis de deuda soberana. Uno de los ajustes más significativos en la materia se dio en el marco del propio Fondo: en las directrices para el otorgamiento de préstamos y en los criterios para la evaluación de la sustentabilidad de las deudas soberanas, concretamente. Lejos en propósito de relevar cada uno de los aspectos involucrados en las reformas referidas ${ }^{15}$, se limitan estas líneas a comentar que en febrero de 2003, esto es, el mismo mes en el que México emitió títulos públicos con CACs, el Comité Ejecutivo del FMI aprobó nuevas directrices para la concesión de préstamos del organismo, las cuales prohibían el otorgamiento de créditos de gran escala a países miembros cuya deuda soberana no se reportase altamente sustentable en el mediano plazo. Dicha decisión, impulsada por Estados Unidos y respaldada por los países Europeos, procuraba atender precisamente el interés de los mencionados actores en torno al ya renombrado problema del riesgo moral. Pero estos criterios, conviene señalar, fueron "relajados" y/o desatendidos en el contexto de la crisis griega a causa de la presión de los países europeos -Alemania y Francia, concretamente- en el directorio del organismo, todo lo cual puso en duda la firmeza de esta reforma.

Por otra parte, el modelo contractualista continuó siendo impulsado por numerosos actores durante la segunda década del siglo XXI al identificarla como la respuesta más inocua en términos de costos políticos y económicos. En este punto, es de acentuar que el abordaje contractualista abrevó en la salida concertada por las autoridades de la Eurozona al resolver incorporar cláusulas de acción colectiva (CAC) en todas las emisiones realizadas por sus Estados miembros con un plazo de madurez mayor a un año y cuyo primer tramo se emitiese después del 1 de enero de $2013^{16}$. Acordada en la cumbre de Ministros de Economía y Finanzas de noviembre de $2010^{17}$ y consagrada en el Tratado del Establecimiento del Mecanismo de Estabilidad Europeo de febrero de 2012 ${ }^{18}$, esta última decisión se constituyó como una respuesta regional a aquellas deficiencias institucionales internacionales en materia de previsión y gestión de crisis de deuda soberana. A tales efectos, se indica que no obstante haber emitido durante años instrumentos de deuda pública nominados en una moneda común, fue recién a partir de la entrada en vigencia de la men- 
cionada disposición que los Estados de la Eurozona se comprometieron a incluir compulsivamente provisiones legales uniformes dentro de los contratos rectores de su endeudamiento soberano, con el objeto de especificar los derechos y limitaciones de los acreedores -y de sí mismos, por contrapartida- en episodios de crisis de deuda soberana, y muy especialmente en los escenarios eventuales de reestructuración (BROOKS y LOMBARDI, 2014).

Conforme los promotores de la iniciativa -Alemania y Francia, en lo principal-, la incorporación de estas cláusulas abriría un horizonte de previsibilidad al enrevesado panorama político-económico europeo puesto que habría de ratificar la determinación de los Estados de la Eurozona de reforzar sus compromisos institucionales en la materia. Por otro lado, estipulaban que la implementación obligatoria de las CACs redundaría en una reducción de los tiempos y costos políticos-judiciales de las reestructuraciones potenciales en la región, al habilitar la modificación de los términos incorporados en los instrumentos de deuda mediante una aprobación de una mayoría calificada de acreedores y limitar, en paralelo a ello, el poder de litigio -y bloqueo, por extensión- de potenciales holdouts, es decir, de aquellos tenedores de títulos públicos renuentes a participar en toda operación de canje. En este marco, y replicando los argumentos centrales de Taylor en la década anterior, los promotores de la iniciativa argumentaron que la incorporación de cláusulas comunes limitaría la necesidad de arbitrar mecanismos de rescate para Estados con problemas de endeudamiento soberano, ya que la superación de la crisis eventual descansaría primariamente en las capacidades de las autoridades estatales para negociar una reestructuración eventual sin comprometer recursos habilitados por ciudadanos de otros Estados. Del mismo modo, y en continuidad con las líneas argumentales de la administración Bush Jr., los promotores del abordaje contractualista en la Eurozona insistieron que la adopción común de las CACs anularía la raíz del "riesgo moral", al constreñirse toda eventualidad de rescate.

Por otra parte, el abordaje contractualista procuró ser reforzado mediante la revisión de la redacción de los contratos de deuda soberana a fin de evitar la repetición de interpretaciones polémicas de las cláusulas en sedes judiciales una vez consumada una crisis, tal cual lo sucedido con la lectura hecha por el juez Thomas Griesa de la cláusula "pari passu" en ciertas demandas contra la República Argentina. En este marco, cobró relevancia la iniciativa del Tesoro norteamericano de organizar un grupo de trabajo de alto nivel. En recurso de la exposición de Brooks y Helleiner, ha de agregarse que "el grupo de trabajo hizo converger a representantes de países en desarrollo y economías emergentes (incluidos Brasil, México, Turquía y Uruguay), Estados del G7 (incluidos Francia, Alemania, el Reino Unido y Estados Unidos), el FMI, la comunidad financiera internacional y una numerosa red de académicos y abogados expertos en deuda. El más significativo de los actores privados que participaron en el proceso fue la Asociación Internacional de Mercado de Capital (ICMA, por sus siglas en español), la cual asumió la responsabilidad de redactar un borrador con los nuevos términos de mercado"19 (BROOKS ; HELLEINER, 2017, p. 1096, traducción propia). El resultado de este trabajo fue
19. En el texto original: "The working group consisted of representatives from developing and emerging market countries (including Brazil, Mexico, Turkey and Uruguay), G7 countries (including France, Germany, the UK and the US), the IMF and the private global financial community, as well as a handful of academics and lawyers with sovereign debt expertise. The most significant private-sector actor in this process was the London-based International Capital Market Associa- tion (ICMA)—a leading financial industry body - which ultimately took respon- sibility for drafting and marketing the new contract terms". 
finalmente publicado en agosto de 2014, recomendándose la implementación de unas "super CACs", las cuales permitan a los Estados avanzar en una renegociación de los términos de los contratos de deuda en términos más favorables que los contenidos en las cláusulas de agregación.

En su intento por escapar del acoso judicial de sus holdouts en jurisdicciones extranjeras -de la norteamericana, en particular-, la República Argentina comenzó a reconsiderar las ventajas del esquema estatutario, el cual había sido oportunamente resistido habida cuenta de la asociación del mismo con el Fondo, un actor con el cual las administraciones nacionales asumidas tras el colapso de principios de siglo habían trabado una relación harto conflictiva. Aunque con un perfil harto diferenciado de aquel auspiciado por Krueger y el FMI a inicios de la década pasada, la iniciativa -de naturaleza preeminentemente reactiva- tomó impulso muy particularmente tras el revés experimentado por la decisión de la Corte Suprema de Estados Unidos de no hacer lugar a un recurso de apelación del país en el que solicitaba la revisión de fallos de primera y segunda instancia que resguardaba la igualdad de trato -bajo la cláusula "pari passu"entre los acreedores que habían aceptado las ofertas de reestructuración de 2005 y 2010 y aquellos que no (Fernández Alonso, 2017).

Llegado a este punto, conviene recordar que las colocaciones realizadas por la República Argentina en sus dos tramos de la reestructuración habían incorporado CACs. Pero ello, deviene imperioso subrayar, no terminó de resolver sus problemas legales con los tenedores de títulos públicos en situación irregular. Más aún, los intensificaron tras la eventualidad expresada por ciertos holdins de activar la arriba mencionada cláusula de aceleración tras las limitaciones de la República Argentina para honrar sus compromisos de pago en Estados Unidos y otras jurisdicciones en instancias del embargo resuelto y ratificado por las instancias judiciales de dicho país en los reclamos de los holdouts respecto al trato equitativo. En este sentido, se expuso cómo tenedores de títulos públicos históricos -esto es, sin CACs- podían afectar la situación de tenedores de títulos públicos nuevos -con CACs- y tras ello, todo un proceso de reestructuración que para entonces ya sumaba el 93\% de aceptación. Al entender de los funcionarios argentinos, esta situación marcaba uno de los aspectos más ventajosos del modelo estatutario frente al contractualista, ya que las CACs no pueden ser impuestas en forma retrospectiva, a menos que se traten de instrumentos regidos por la ley del propio Estado, tal cual lo ocurrido en Grecia en 2012.

Así pues, la República Argentina trabó gestiones con otros Estados en desarrollo en aras de mocionar una resolución en la Asamblea General de Naciones Unidas (AGNU o UNGA, por sus siglas en inglés) conducente a establecer "un marco jurídico multilateral para los procesos de reestructuración de la deuda soberana con miras a, entre otras cosas, aumentar la eficiencia, la estabilidad y la previsibilidad del sistema financiero internacional, y lograr un crecimiento económico sostenido, inclusivo y equitativo y el desarrollo sostenible, de conformidad con las circunstancias y prioridades nacionales" (AGNU, 2014).

Como bien detalla Alejandro Manzo (2018), la propuesta de la República Argentina se inscribió como el resultado de un trabajo de larga data en el marco de la Conferencia de Naciones Unidas para el Comercio 
y el Desarrollo (UNCTAD). Detractora de las capacidades autorreguladoras de los mercados, la propuesta de la República Argentina sustentaba una perspectiva propia de la relación Estado-mercado, en la que debía primar el primer elemento. Así pues, a diferencia de la propuesta estatutaria del FMI de principios de siglo que procuraba reubicar el rol del organismo en la escena económica internacional, la formulada por la República Argentina tuvo una vocación más universalista y democrática, ya que las reglas que conducirían los procesos de reestructuración de deuda soberana serían canalizados conforme los principios aprobados por la Asamblea General, y no por una instancia cuyas decisiones son fruto de un proceso poco transparente y con voto ponderado.

De la misma manera, la iniciativa argentina procuraba la participación activa de la comunidad internacional mediante la conformación de una Comisión Observadora -conformada ad hoc por tres Estados (..). Entre otras facultades, la iniciativa le otorga a la Comisión la capacidad para estudiar la información presentada por el Estado al tiempo de activar el proceso, resolver disputas y redactar un informe final mostrando si el deudor soberano ha cumplido o no con los preceptos del mecanismo regulatorio propuesto" (MANZO, 2018, p. 28).

Por otra parte, la propuesta de la República Argentina en Naciones Unidas (NU) procuró afirmar el principio de la autonomía de los Estados para diseñar y ejecutar los procesos de reestructuración sin condicionamientos de terceros, sean organismos multilaterales de crédito, gobiernos nacionales o agentes privados. Dicho esto, puede indicarse que el proyecto de la República Argentina no se preocupaba del problema del riesgo moral al concebir, en última instancia, que los mercados no operan necesariamente de modo eficiente y que los efectos a considerar tras la provisión de un paquete de rescate son más bien sobre los ciudadanos del país en crisis que sobre el sistema financiero en general.

En relación con lo antedicho, y siguiendo a Manzo (2018), es dable apuntar que el proyecto de la República Argentina entendía el principio de sostenibilidad no sólo en términos de sustentabilidad de la deuda soberna; sino que también (o más bien) en función del desarrollo. Al respecto, y tal cual marcó la resolución aprobada, las reestructuraciones "deben promover el crecimiento económico sostenido e inclusivo y al desarrollo sostenible, minimizando los costos económicos y sociales, garantizando la estabilidad del sistema financiero internacional y respetando los Derechos Humanos" (A/RES/69/319 2015). De ahí pues, que la preocupación por los ciudadanos que son llamados a pagar los rescates estuviera invertido en relación con las propuestas precedentes. Conforme las ideas fuerza de la propuesta, los costos de las crisis de deuda soberana son distribuidos en la mayor parte de los casos en forma asimétrica, siendo los ciudadanos de los Estados en crisis los más perjudicados.

Por último, en relación con el problema de los holdouts, el proyecto marcaba la necesidad de suspender todos los litigios iniciados en aras de resguardar otro de los principios que propugna el proyecto: la buena fe de todos los afectados por las negociaciones de reestructuración de la deuda.

Habida cuenta de la no obligatoriedad de las resoluciones de la AGNU, los "Principios Básicos de los Procesos de Reestructuración de la 
20. En el texto original: "...CACs will be slow to become effective and will not fully shield sovereigns from legal action. First, it takes many years until a new type of bond contract, such as those with aggregation CACs, disseminates through the outstanding debt stock via new issuances. Second, CACs are no safeguard against litigation either in case investors focus on creating blocking minorities in individual bond series or if they litigate based on other defaulted debts, such as loans".
Deuda Soberana" aprobados por la precitada resolución no imputaron un impacto significativo al régimen de mercado articulado por defecto en 2003 mediante la generalización de las CACs. La iniciativa, apoyada por el G77 más la República Popular China (RPC) contó con la férrea oposición de Estados Unidos y un número significativo de potencias desarrolladas. En este caso, no sólo se manifestaba un rechazo a una iniciativa delegativa más, sino a una encarada cual disruptiva del (des)orden financiero internacional estructurado desde el colapso del orden de Bretton Woods en los setenta y profundizado tras el expandir e intensificar del proceso globalizador. Nada facilitó a subvertir tal situación, la determinación de la administración de Mauricio Macri (2015, en adelante) de discontinuar impulsando la iniciativa pergeñada por su predecesora -Cristina Fernández (2007-2015)- a raíz del diagnóstico contrapuesto del mandatario en relación con la problemática de la deuda soberana argentina.

Llegado a este punto, puede colegirse que el régimen de gestión de crisis de deuda soberana continúa descansando en la lógica contractual, liberal, de mercado asentada a partir de 2003. Tal como advierte el FMI (2016), en el período 2014-2016, aproximadamente el 75\% de las nuevas colocaciones de deuda soberana habían incorporado el nuevo modelo de las CACs. Sin embargo, y siguiendo las formulaciones de Julian Schumacher, Henrik Enderlein y Christoph Trebesch se subraya que todas estas innovaciones asociadas a la incorporación de las CACs "tardarán en hacerse efectivas y no protegerán completamente a los soberanos de demandas legales. Primeramente, debido a que debe transcurrir mucho tiempo hasta que un nuevo tipo de contrato de bonos, tal como aquellos que tienen CACs de agregación, se disemine alresto de la deuda ya emitida a través de nuevas colocaciones. En segundo lugar, las CAC no son una salvaguarda contra los litigios, ya sea en caso de que los inversores se centren en crear minorías bloqueadoras en series de bonos o si litigan en base a otras deudas incumplidas, tales como préstamos"20 (SCHUMACHER et. al., 2018, p. 53-54, traducción propia).

Reflexiones finales

De gravitación central en la agenda de la economía y la política internacional en los primeros años del siglo XXI, el debate en torno a los esquemas de reestructuración de deuda soberana pasó a un estadio aletargado tras la imposición de facto del esquema contractual en 2003. No obstante, lo que supuso una clausura del debatetras la retirada del proyecto estatutario propuesto por el staff del FMI y la emisión creciente de instrumentos de títulos de deuda soberana con CACs no implicó una solución definitiva. En razón de ello, el estallido de crisis de deuda soberanas en la Eurozona y ciertas economías de la periferia global y los reveses judiciales de la República Argentina en las demandas entabladas en la justicia norteamericana por sus acreedores en situación irregularrehabilitaron las discusiones sobre el particular. Tal como se advirtió en el transcurrir del artículo, los promotores de cada propuesta fueron cambiando de referentes, aunque no de los basamentos sobre los que erigen sus argumentaciones político-económicas. Las propuestas en pugna 
impulsan procedimientos contrapuestos en términos de delegación para prevenir y gestionar crisis de deuda soberana.

A semejanza de lo ocurrido una década precedente, las distancias entre las propuestas continúa latente, reproduciéndose consecuentemente proyectos entre aquellos defensores de una lógica de reestructuración más cercana de mercado y otra de corte más formal. La ausencia de consenso conocida una década atrás, continúa pues en los días presentes.

Mientras tanto, la ausencia de un marco específico para la reestructuración de las deudas soberanas constriñó -y constriñe- las posibilidades de encontrar soluciones prontas, eficientes y sustentables en episodios de crisis de deuda soberana y de defaults, en particular. Este déficit institucional del sistema financiero internacional contemporáneo no impidió, por cierto, que se desplegaran las operaciones en el mismo. Empero, sí afectó a aquellos Estados caídos en crisis, que vieron restringido su acceso al financiamiento internacional y reconocerse crecientemente condicionados a diseñar y ejecutar políticas de ajuste las cuales terminan por atentar contra derechos políticos, económicos y sociales de sus propios ciudadanos. En este contexto, los Estados afectados por una crisis de deuda soberana "pierden crecientemente capacidad para garantizar las necesidades básicas de sus ciudadanos y de salvaguardar sus derechos humanos"21 (GELPERN, 2016, p. 46, traducción propia).

Como oportunamente plantean Martin Guzmán y Joseph Stiglitz, "la ausencia de un régimen para administrar crisis de deuda soberanas conduce a ineficiencias ex-ante y ex-post del estallido de la crisis, y a inequidades tanto entre acreedores como así también entre el deudor y sus acreedores. Adicionalmente, a diferencia de las bancarrotas domésticas, las negociaciones de reestructuración de deudas soberana tienen lugar en un escenario legal ambiguo. Varias jurisdicciones diferentes, todas con diferentes perspectivas, intervienen en el proceso. Diferentes órdenes legales a menudo llegan a conclusiones diferentes para el mismo problema. Puede no estar claro cuál prevalecerá (y posiblemente ninguno de ellos prevalecerá), y cómo se resolverá la negociación implícita entre los poderes judiciales de los diferentes países" (GUZMÁN; STIGLITZ, 2016, p. 4).

El crecimiento de los niveles de deuda a nivel mundial alimentado por la gran liquidez internacional durantelos últimos años invita a pensar que los episodios de crisis de deuda soberana se repetirán con más frecuencia en el futuro, poniendo en evidencia las externalidades negativas del mencionado déficit institucional internacional.

Haciendo propias las palabras de Michael Dooley (2000), se sostiene que un régimen internacionalde reestructuración de la deuda soberana debería no sóloreinsertar a un país devenido en crisis en los mercados financieros, sino que también debería abordar el problema de la deuda soberana de manera temprana y proactiva. En este respecto, y en continuidad con lo formulado por Gitlin y House, dicho régimen de reestructuración de deuda soberana debería "evaluar la solvencia de un país de manera abierta, creíble y directa; alcanzar una visión equilibrada y ampliamente compartida sobre la capacidad del país para pagar su deuda existente; crear las bases para un consenso sobre una distribución razonable de las pérdidas entre un deudor, sus ciudadanos y sus acreedores; $y$
21. En el texto original: "Governments lose their capacity to meet the basic human needs of their citizens and to safeguard their human rights". 
22. En el texto original: "It should address sovereign debt distress early and proactively. It should assess a country's solvency in an open, credible and straightforward fashion; it should reach a balanced, broadly shared view on the country's capacity to pay its existing debt; it should create the foundations for a consensus on a reasonable distribution of losses among a debtor, its citizens and its creditors; and it should set a country back on a path to growth and liquidity with the smallest possible ef ciency losses to stakeholders" volver a colocar a un país en el camino hacia el crecimiento y la liquidez con las menores pérdidas de eficiencia posibles para las partes interesadas"22 (GITLIN ; HOUSE, 2015, p. 11, traducción propia).

Por todo lo antedicho, resulta más pertinente que los responsables de saldar las deficiencias institucionales ensayen respuestas alternativas a una problemática tan compleja como la resolución de crisis de deuda soberana. El desafío, desde luego, no es nimio. A la luz de la experiencia de los primeros años del siglo, el arreglo institucional que aspire saldar el mentado déficit normativo en la materia debeidentificar el equilibrio entre los beneficios derivados de un régimen internacional de gestión de deuda soberana claro y previsible y los costos eventuales identificados por aquellos actores renuentes a una empresa delegativa, Estados Unidos y los principales actores del sistema financiero internacional, en breve.

Referencias

ABBOTT, K., KEOHANE, R., MORAVCSIK, A., SLAUGHTER, A; DUNCAN, S., The Concept of Legalization. En: GOLDSTEIN, J., KAHLER, M., KEOHANE, R; SLAUGHTER, A. Legalization and World Politics International Organization Special Issues, Cambridge, MA: The MIT Press, 2000

AKYÜZ, Y. (ed.), Reforming the Global Financial Architecture. Issues and Proposals, Londres,UNCTAD-TWN-Zed Books, 2002.

ALFARO, C. Sovereign Insolvency and Debt Restructuring, Journal of Corporate Renewal, 2003.

ASAMBLEA GENERAL DE NACIONES UNIDAS (UNGA), Hacia el establecimiento de un marco jurídico multilateral para los procesos de reestructuración de la deuda soberana. A/68/L.57/ Rev.1, 2014.

ASAMBLEA GENERAL DE NACIONES UNIDAS (UNGA) "Principios Básicos de los Procesos de Reestructuración de la Deuda Soberana”. A/69/L.84, 2015.

BUCHHEIT, L.; GELPERN, A.; GULATI, M; PANIZZA, U.; WEDER DI MAURO, B.; ZETTELMEYER, J. Revisiting Sovereign Bankruptcy, Committee on International Economic Policy and Reform, Washington: Brookings, 2013. Disponible en: https://www.brookings.edu/wp content/uploads/2016/06/CIEPR_2013_RevisitingSovereignBankruptcyReport.pdf> Última consulta en: 30 abr. 2018.

BUCHIERI, F. «Default soberanos: la necesidad de reforma a la arquitectura financiera internacional a partir de la experiencia de Argentina, Ciencias Económicas, v. 11 n. 02, 2014

BUSTELO, P. La construcción de una nueva arquitectura financiera internacional: progresos y propuestas, Boletín Económico de ICE, n. 2642, 31 de enero-13 de febrero, 2000, p. 25- 33.

BUSTELO, P. La Nueva Arquitectura Financiera Internacional, Cuadernos de la Escuela Diplomática, n. 26, 2005, p. 153-181.

BROOKS, S; HELLEINER, E.«Debtpolitics as usual? Reforming the sovereign debt restructuring regime after 2008.,International Affairs, v. 93, n. 5, 2017. p. 1085-1105.

BROOKS, S.; LOMBARDI, D. (2014), «Sovereign Debt Restructuring: A Backgrounder, Issues Paper for the Global Consultations on Sovereign Debt Restructuring, New Rules for Global Finance, Washington DC, 2014.Disponible en: http://www.new-rules.org/storage/documents/sovereign_debt_restructuring_background_paper_draft2014.pdf. Última consulta en: 13 sept. 2016.

CHUKWU, F.Sovereign Debt Restructuring: Lessons from Argentina, Journal of Politics \& International Affairs, Spring, v. 8, 2011.

COSIO-PASCAL, E. The Emerging of a Multilateral Forum for Debt Restructuring: The Paris Club, UNCTAD Discussion Papers, n. 192, 2008. Disponible en: http://unctad.org/en/Docs/ osgdp20087_en.pdf. Última consulta en: 05 out 2017.

COUILLAULT, B., y WEBER, P. Toward a Voluntary Code of Good Conduct for Sovereign Debt Restructuring, Financial Stability Review, Banque de France, Paris, 2003.

CRUCES, J. J. Una república sin buitres, Buenos Aires: Fondo de Cultura Económica (FCE), 2016. 
DAS, U., PAPAIOANNOU, M; TREBESCH, Ch. Sovereign Debt Restructurings 1950-2010: Literature Survey, Data, and Stylized Facts, Working Paper, 2012. Disponible en https://www.imf. org/external/pubs/ft/wp/2012/wp12203.pdf. Úlltima consulta en: 25 out. 2017.

DOBBS, R., LUND, S.,WOETZEL, J; MUTAFCHIEVA, M. Debt and (not much) deleveraging, Washington, DC: McKinsey Global Institute, 2015. Disponible en: www.mckinsey.com/insights/economic_studies/debt_and_not_much_deleveraging. Última consulta en: 3 may. 2018.

EICHENGREEN, B. Restructuring Sovereign Debt, Journal of Economic Perspectives, n.17,v.3, 2003.

EUROPEAN UNION. Statement by the Eurogroup, November 28, 2010. Disponible en: http:// www.consilium.europa.eu/press/press-releases/2010/11/pdf/Statement-by-the-Eurogroup/. Última consulta en: 17 dez. 2017.

FERNÁNDEZ ALONSO, José. La cuestión de la deudasoberanaen la política exterior argentina desde la vuelta a la democracia. En: BUSSO, Anabella (Coord.), Política Exterior Argentina y Modelos de Desarrollo. Aportes para el análisis de la inserción internacional desde la redemocratización, v. 2, Rosario: UNR Editora, 2017, p. 164-192.

FONDO MONETARIO INTERNACIONAL. Second Progress Report on Inclusion of Enhanced Contractual Provisions in International Sovereign Bond Contracts. Washington: IMF, 2016.

FONDO MONETARIO INTERNACIONAL. Monitor Fiscal,,Washington:IM, 2018. Disponible en::http://www.imf.org/es/Publications/FM/Issues/2018/04/06/fiscal-monitor-april-2018. Última consulta en: 19 mai. 2018.

GALLAGHER, K. The New Vulture Culture: Sovereign debt restructuring and trade and investment treaties. IDEAs Working Paper, n. 02, 2011.

GELPERN, A. A Skeptic’s Case for Sovereign Bankruptcy, Houston Law Review, v. 50, 2013

GELPERN, A.Sovereign Debt: Now What? The Yale Journal of International Law, v.. 41, n. 2. , 2016, p. 45-85.

GELPERN, A., HELLER, B, y SETSER, B. Count the limbs: designing robust aggregation clauses in sovereign bonds. En: GUZMAN, M., OCAMPO, J.A. y STIGLITZ, J. (eds), Too little, too late, New York: Columbia University Press, 2016.

GIANVITI, F. et al (2010), European Mechanism for Sovereign Debt Crisis Resolution: A Proposal, Bruegel, 2010. Disponible en: http://www.bruegel.org/publications/publication-detail/ publication/446-a-european-mechanism-for-sovereign-debt-crisis-resolution-a-proposal.Última consulta en: 27 out. 2016.

GITLIN, R; HOUSE, B. Just Enough, Just in Time: Improving Sovereign Debt Restructuring for Creditors, Debtors and Citizens, Special Report, Centre for International Governance Innovatio, Ontario, 2015. Disponible en: https://www.cigionline.org/sites/default/files/just_in_time_ special_report_0.pdf. Última consulta en: 4 abr.2018.

GOLDSTEIN, J., KAHLER, M., KEOHANE, R; SLAUGHTER, A Introduction: Legalization and World Politics. En: GOLDSTEIN, J., KAHLER, M., KEOHANE, R; SLAUGHTER, A. Legalization and World Politics International Organization Special Issues, Cambridge, MA: The MIT Press, 2001

GUZMÁN, M.; STIGLITZ, J. Creating a framework for sovereign debt restructuring that works. En: GUZMÁN, M.; OCAMPO, J.A.;STIGLITZ, J. (Eds.) Too Little, Too Late: The Quest to Resolve Sovereign Debt Crises, New York: Columbia UniversityPress, 2016.

HALEY, J. Sovereing Debt Restructuring: Old Debates, New Challenges, CIGI Papers, Ontario, n. 32,2014

HELLEINER, E.The strange story of Bush and the Argentine debt crisis. En: Third World Quarterly, v. 26, n. 6, 2005,p. 951-969.

HOFFMAN, C. Sovereign-Debt Restructuring in Europe Under the New Model Collective Action Clauses, Texas International Law Journal, v. 49, 2014, p. 383-445.

INSTITUTE FOR INTERNATIONAL FINANCE (IIF). Principles for Stable Capital Flows and Fair Debt Restructuring in Emerging Markets, Washington, DC, 2014.

KINDLEBERGER, Ch. Manias, Panics, and Crashes, Basic Books, New York, 1978.

KRASNER, S. Conflicto Estructural: El Tercer Mundo Contra el Liberalismo Global, Buenos Aires, Grupo de Editores Latinoamericanos, 1989.

KUEGER, A. A New Approach To Sovereign Debt Restructuring, International Monetary Fond, Washington, 2002 
LI, Y. Question the Unquestionable Beauty of a Collective Proceeding for All Sovereign Debt Claims. Annual Meeting of the Academic Forum of INSOL International, Trier, Alemania, 2013. Disponible en: http://ssrn.com/abstract=2234210. Última consulta en: 5 out. 2010.

LIPSON, Ch. Standing Guard. Protecting Foreign Capital in the Ninetheenth and Twentieth Centuries, Berkeley, University of California Press, 1985.

MANZO, A. Reestructuraciones de deuda soberana: El debate sobre su regulación en términos de disputa por la gobernanza global». Revista Direito e Práxis, v. 9, n.1, 2018, p.9-45.

OCAMPO, J. A. Reforma de la arquitectura monetaria y financiera internacional, Dialogue on Globalization, Friedrich-Ebert-Stiftung, November, 2014. Disponible en: http://www.nuso. org/upload/articulos/ANALISIS\%20Reforma\%20de\%20la\%20arquitectura\%20monetaria.pdf. Última consulta en: 16 out. 2016.

PANIZZA, Ugo. Do we need a mechanism for solving sovereign debt crises? A rule-based discussion, Graduate Institute of International and Development Studies Working Paper, Ginebra: GraduateInstitute of International and Development Studies, n. 3,2013,

REIFFEL, L. Restructuring Sovereign Debt: The Case for Ad Hoc Machinery, Washington DC, Brookings Institution Press, 2013.

REINISCH, A. Ein Insolvenzrecht für Staaten?. Wirtschaftspolitische Blätter, n. 50, 2003, p. 285-289.

ROUBINI, N.; SETSER, B. The reform of the Sovereign Debt Restructuring Process: Problems, Proposed Solutions and the Argentine Episode", Journal of Restructuring Finance, v.1, n.1, 2004, p. 173-184.

SCHUMACHER, J.; ENDERLEIN, H.; TREBESCH, Ch. Sovereign defaults in court, ECB Working Paper, Frankfurt: European Central Bank (ECB), n. 2135, 2018.

SCHWARCZ, S. Idiot's Guide to Sovereign Debt Restructuring. Emory LJ, v.53, n.1189, 2004.

STANLEY, L. Resolución de conflictos en material de bonos soberanos: una "crítica" mirada institucional al papel del CIADI. Nuevos Documentos del CEDES, Buenos Aires, n.58, 2009.

STIGLITZ, J. Odious Rulers, Odious Debts, Atlantic Monthly, November, 2013. Disponibe en: http://www.theatlantic.com/magazine/archive/2003/11/odious-rulers-odious-debts/302831/. Última consulta en: 2 nov. 2016.

STURZENEGGER, F. y ZETTELMEYER, J. Debt Defaults and Lessons from a Decade of Crises, Massachusetts,The MIT Press, 2006.

TAMURA, K. The problem of sovereign debt restructuring: How can we deal with Holdout problem legally? International Finance Seminar Professors Hal Scott and Howell Jackson, Harvard Law School, Boston, 2002. Disponible en: http://www.law.harvard.edu/programs/ about/pifs/education/1lm/2001---2002/sp45.pdf Última consulta en: 1 nov. 2016.

TOMZ, M. Reputation and International Cooperation. Sovereign Debt across Three Centuries, New Jersey, Princeton, 2007.

UGARTECHE, O.; ACOSTA, A.Post Bretton Woods: los problemas de la economía global, y el tribunal internacional de arbitraje de deuda soberana. En: ACOSTA, A. ; FALCONI, F. Asedios a lo imposible. Propuestas económicas en construcción, FLACSO - ILDIS - FES, Quito, 2005, p. 223-264.

UNDERHILL, G; X. ZHANG (eds.) International Financial Governance Under Stress. Global Structures versus National Imperatives, Cambridge: Cambridge University Press, 2003.

VILLANUEVA, J. El debate de la Nueva Arquitectura Internacional Financiera, Serie Seminarios, Instituto y Universidad Torcuato Di Tella, Buenos Aires, 2003.

WAIBEL, M. Sovereign Defaults before International Courts and Tribunals, Cambridge University Press, Cambridge, 2011.

WINKLER, M. Foreign bonds: An autopsy. Philadelphia: Roland Swain, New York, Arno Press, 1976.

WRIGHT, M. Sovereign Debt Restructuring: Problems and Prospects, Harvard Business Law Review, v. 2, 2012, p. 154-197. 